\title{
Amorphization of Molecular Liquids of Pharmaceutical Drugs by Acoustic Levitation
}

\author{
C. J. Benmore and J. K. R. Weber \\ X-ray Science Division, Advanced Photon Source, Argonne National Laboratory, Argonne, Illinois 60439, USA
}

(Received 22 February 2011; revised manuscript received 17 May 2011; published 8 August 2011)

\begin{abstract}
It is demonstrated that acoustic levitation is able to produce amorphous forms from a variety of organic molecular compounds with different glass forming abilities. This can lead to enhanced solubility for pharmaceutical applications. High-energy x-ray experiments show that several viscous gels form from saturated pharmaceutical drug solutions after 10-20 min of levitation at room temperature, most of which can be frozen in solid form. Laser heating of ultrasonically levitated drugs can also result in the vitrification of molecular liquids, which is not attainable using conventional amorphization methods.
\end{abstract}

DOI: 10.1103/PhysRevX.1.011004 Subject Areas: Interdisciplinary Physics, Materials Science, Medical Physics

\section{INTRODUCTION}

Because of increased surface wetting of their disordered structure, changes in molecular conformation, or intermolecular bonding, drugs in solid but amorphous forms often have enhanced solubility, faster dissolution rates, and higher bioavailability compared to their crystalline counterparts [1-6]. A limited range of amorphous pharmaceutical compounds are already commercially available but synthesizing bulk amorphous forms of a drug is not always possible using conventional pharmaceutical processes such as melt quenching in containers, freeze and spray drying, ball milling, desolvation, or precipitation methods [1-5]. In this paper we have employed an acoustic levitation method $[7,8]$ to amorphize, at or above room temperature, a wide range of organic molecular liquids and solutions that are relevant to the pharmaceutical industry. Furthermore, highenergy $\mathrm{x}$-ray diffraction has been used to characterize and monitor changes in the bulk structure of several drug solutions suspended in an acoustic levitator over time and of glassy drugs produced from laser heated, levitated droplets.

In recent years, aerodynamic, electromagnetic, and electrostatic levitation techniques have become increasingly popular in the investigation of high temperature oxide and metallic melts [8,9]. In many cases these containerless techniques have been shown to supercool high temperature melts several hundred degrees below their melting temperatures [8-10]. The acoustic levitation technique, normally confined to microgravity experiments [7], allows ca. 1-3 mm diameter droplets to float freely without coming into contact with a surface (see Fig. 1). Since containerless processing avoids extrinsic heterogeneous nucleation by surface contact that would normally occur in containers, it often enables liquids to be supercooled or supersaturated

Published by the American Physical Society under the terms of the Creative Commons Attribution 3.0 License. Further distribution of this work must maintain attribution to the author(s) and the published article's title, journal citation, and DOI. well beyond equilibrium conditions. The acoustic streaming also causes additional forced convective cooling that increases the cooling rate slightly (by a few degrees/ second). The ultrasonic levitation technique isolates reactive materials, inhibits contamination from impurities, and is found in this work to amorphize a variety of molecular liquids. Here we define the term amorphous to mean that the solid material does not exhibit diffraction Bragg peaks that result from a repeating, long-range periodic atomic or molecular ordering [11].

There have only been a few previous studies using acoustic levitation to investigate biological materials. Varanasi et al. [12] used the device to characterize the properties of lipids, and Chung and Trinh [13] used a combined ultrasonic-electrostatic levitator as a method for growing protein crystals in a containerless environment. More recently, the crystallization of aspirin and vitamin $\mathrm{C}$ has been followed through the evaporation of an aqueous droplet held in an acoustic levitator using synchrotron diffraction by Leiterer et al. [14]. However, to our knowledge this is the first time that acoustic containerless processing has been used to produce amorphous solids of organic materials. We have found that the containerless processing technique is useful for producing new solution-derived amorphous forms of drugs and glassy forms difficult to vitrify by quenching from the melt. We show that the method can be applied to a wide range of chemical structures with varying pharmaceutical uses.

\section{EXPERIMENTAL}

Containerless methods are described below for producing amorphous forms of several drugs with a variety of chemical structures and different functions by two routes, namely, solvent evaporation and laser heating. The starting materials were purchased from SigmaAldrich (see Table I) and used without further purification. For the evaporation experiments drug materials were selected, which showed some propensity for forming viscous fluids before crystallizing when left to dry in a container during an initial screening procedure. The crystalline 
TABLE I. Details of the starting materials that were purchased from Sigma-Aldrich. Also shown are some physical properties taken from Sigma-Aldrich (*), Baird et al. [15], and Dudognon et al. for racemic Ibuprofen [16].

\begin{tabular}{|c|c|c|c|c|c|c|c|}
\hline Material & CAS number & $\begin{array}{l}\text { Catalogue } \\
\text { number }\end{array}$ & $\begin{array}{c}\text { Lot } \\
\text { number }\end{array}$ & Purity (\%) & $\begin{array}{c}\text { Melting } \\
\text { temperature }\left({ }^{\circ} \mathrm{C}\right)\end{array}$ & $\begin{array}{l}\text { Glass transition } \\
\text { temperature }\left({ }^{\circ} \mathrm{C}\right)\end{array}$ & $\begin{array}{l}\text { Description of levitated } \\
\text { melt quenched } \\
\text { product }\end{array}$ \\
\hline Clotrimazole & $23593-75-1$ & C6019 & $075 \mathrm{~K} 1032$ & 99 & $143-144 *$ & $30[15]$ & Clear solid \\
\hline Probucol & $23288-49-5$ & P9672 & 079K1589 & 100 & $127[15]$ & $27[15]$ & Clear flakes, brittle solid \\
\hline $\begin{array}{l}\text { Ibuprofen } \\
\text { (racemic) }\end{array}$ & $15687-27-1$ & I7905 & $078 \mathrm{H} 4621$ & 98 & $76[16]$ & $-45[16]$ & $\cdots$ \\
\hline Dibucaine & $85-79-0$ & D0513 & $049 \mathrm{~K} 1165 \mathrm{~V}$ & 99 & $65[15]$ & $-35[15]$ & $\begin{array}{l}\text { Clear viscous drop, } \\
\text { turned white in minutes }\end{array}$ \\
\hline Ketoprofen & $22071-15-4$ & K1751 & BCBC1688 & 98 & $95[15]$ & $-3[15]$ & Clear viscous solid \\
\hline Clofoctol & $37693-01-9$ & C2290 & $018 \mathrm{~F} 0644$ & $>99$ & 88 [15] & $-4[15]$ & Clear viscous solid \\
\hline Cinnarizine & 298-57-7 & C5270 & 099K1071 & $>99$ & $121[15]$ & 7 [15] & $\begin{array}{l}\text { Clear viscous solid } \\
\text { with a yellow tint }\end{array}$ \\
\hline Carbamazepine & 298-46-4 & C4024 & $128 \mathrm{~K} 1261$ & 100 & $192 *$ & $61[15]$ & Clear solid \\
\hline $\begin{array}{l}\text { Miconazole } \\
\text { Nitrate }\end{array}$ & $22832-87-7$ & M3512 & 058K1301 & 100 & $86[15]$ & 1 [15] & Dark amber solid \\
\hline
\end{tabular}

pharmaceutical drugs Clotrimazole, Ibuprofen, Dibucaine, Ketoprofen, and Clofoctol were dissolved in pure anhydrous ethanol and Probucol was dissolved in acetone. The solvents were chosen because of the high solubility of the compounds. Saturated solutions were prepared by adding material to the solvent that was held in a glass vial in an ultrasonic mixing bath and the resulting solutions were filtered to remove undissolved solids. Weighed samples of each solution were dried out to determine the solute concentration. Droplets of the solutions were introduced into the acoustic levitator, which was operating at a frequency of $22 \mathrm{kHz}$ using a clean $1 \mathrm{ml}$ syringe. This instrumentation has been described in detail in a previous publication [17] and the process for this application is illustrated in Fig. 1(a).

The structure of the starting crystalline materials, the amorphous samples processed in the levitator, and the progress of the evaporation experiments were measured using the high-energy $\mathrm{x}$-ray diffraction beamline 11-ID-C at the Advanced Photon Source. High-energy synchrotron $115 \mathrm{keV} \mathrm{x}$-rays are a bulk probe and, therefore, are highly sensitive to the degree of glass formation. Combined with the very high statistical accuracy, we estimate that this technique determines the amount of amorphous material to within $\sim 0.2 \%$, compared to a few percent using a laboratory x-ray source. In the experiments reported here, the x-rays had an incident wavelength of $0.10798 \AA$ $(\sim 115 \mathrm{keV})$ and the scattered $\mathrm{x}$-ray intensity was measured with a flat plate a-Si Perkin Elmer area detector (model XRD 1621). The method enabled the structural evolution of the bulk material to be monitored over time and has been described previously [18]. The data were acquired from levitated samples in 2 min duration sets. The in situ measurements were taken for periods of up to 20 min for most samples, although the aging and drying of the amorphous gels were monitored for up to $10 \mathrm{hr}$ for Probucol and $14 \mathrm{hr}$ for Ibuprofen. The 2D detector data were corrected for variations in dark current at least every $20 \mathrm{~min}$ and the sample to detector distance was determined by levitating a polystyrene ball coated in polycrystalline $\mathrm{CeO}_{2}$ powder. The 2D sample data were corrected for background, beam polarization, detector geometry, and integrated to 1D using the software FIT2D [19]. The x-ray structure factors, $S(Q)$, were obtained up to a maximum $Q \sim 20 \AA^{-1}$ using the program PDFGETX2 [20], which

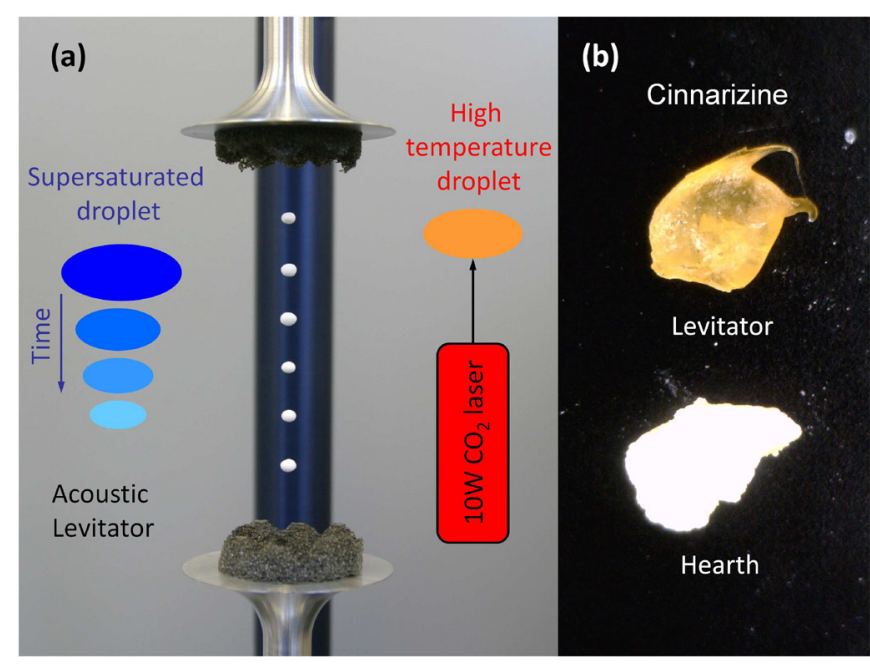

FIG. 1. (a) Photograph of the acoustic levitator levitating several samples simultaneously (shown as white spheres) with a $14 \mathrm{~mm}$ spacing. The illustrations on either side of the levitator show the two methods of forming amorphous forms of pharmaceutical drugs in this study. (b) A photograph of pure Cinnarizine melted in the levitator to form a yellow amorphous product, compared to the same starting material melted in the laser hearth that formed a white crystalline product. 


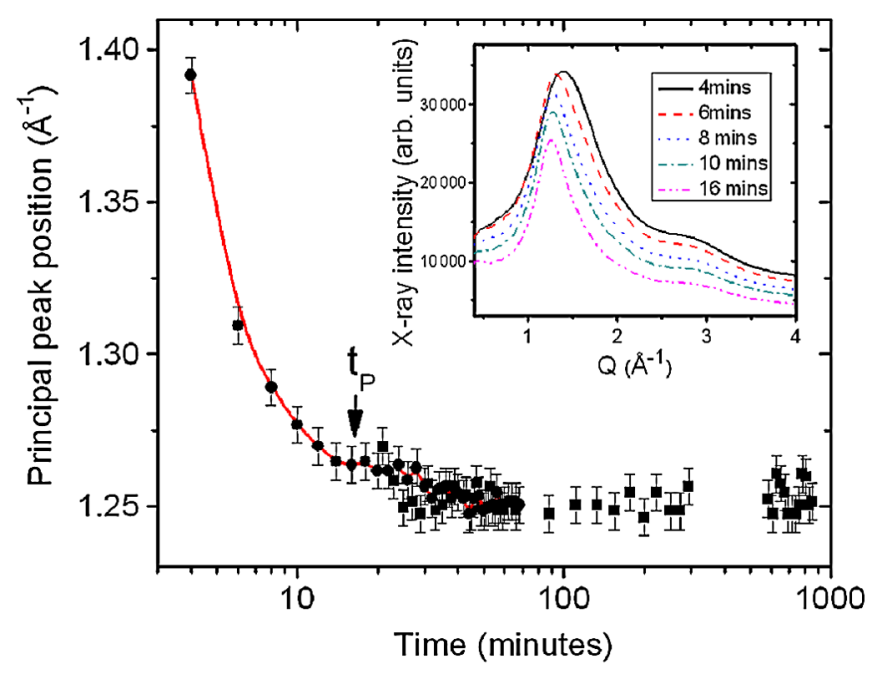

FIG. 2. Insert: The $\mathrm{x}$-ray intensity varying over time as the solvent evaporates from a supersaturated droplet of Ibuprofen in ethanol. Main: The position of the principal peak followed as a function of $\log$. time. The parameter $t_{\mathrm{P}}$ indicates the time at which the position starts to plateau and a viscous amorphous gel starts to form. After the plateau is reached in $\sim 20$ minutes, only minimal changes in structure were observed even after $>14 \mathrm{hr}$ of in situ measurements on the levitated drop.

performs corrections for oblique incidence, energy dependent detector efficiency absorption, and multiple scattering. The concentrated solution and time-resolved changes leading to the formation of an amorphous gel for Ibuprofen in ethanol are illustrated in Fig. 2, where the position of the first peak in the structure factor is shown as a function of time. The plateau in the positions was found to correspond to an amorphous gel form. The drying of all the pharmaceutical solutions in containers resulted in crystalline products.

\section{RESULTS AND DISCUSSION}

\section{A. Amorphization from solutions}

Once the liquids were levitated, the solvent started to evaporate because its vapor pressure is much higher than that of the solute molecules. Since acoustic levitation avoids the nucleation of crystals that frequently occurs at the interface between a liquid and a solid surface, the solutions were easily supersaturated. After a period of 10 to $20 \mathrm{~min}$ in the levitator, the concentration of solute increased sufficiently for the liquids to become extremely viscous, resulting in amorphous gels. The diffraction patterns of these gels were observed to be significantly different from that of the pure solvent, showing that most of the bulk solvent had evaporated (see Fig. 3). Given the viscous nature of the gels, this suggested that, in most cases, some of the solvent had interacted (via hydrogen bonding or other interactions) with the drug, such that only the bulk solvent was evaporated. This leads to the increased

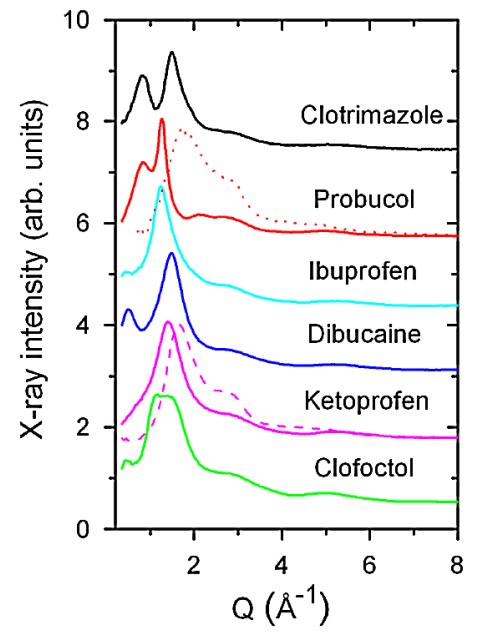

FIG. 3. The background corrected x-ray diffraction patterns for six amorphous gels formed from supersaturated solutions measured in situ using the acoustic levitator. These x-ray spectra were taken after $14 \mathrm{~min}$ of levitation for Clotrimazole, after $20 \mathrm{~min}$ for Probucol, after $16 \mathrm{~min}$ for Ibuprofen, after $10 \mathrm{~min}$ for Dibucaine, after $16 \mathrm{~min}$ for Ketoprofen, and after $21 \mathrm{~min}$ for Clofoctol. The broken lines represent the x-ray diffraction pattern of the pure solvents used (see text): ethanol (dashed line) and acetone (dotted line).

likelihood of the drug molecules changing their conformation and/or packing arrangements.

By following the position of the principal peak in the $\mathrm{x}$-ray diffraction patterns for all the supersaturated solutions it was possible to track the amorphization process as a function of time. The principal peak in the x-ray diffraction pattern of pure ethanol is known to be dominated by intermolecular carbon and oxygen interations [21], and this peak moves to lower- $Q$ values as the solvent evaporates from the Ibuprofen solution data shown in Fig. 2. The peak in the final spectra is therefore assigned to intra- and/ or intermolecular interactions associated with the drug molecules. We observed that as the principal peak position reached the beginning of a plateau at time $t_{\mathrm{P}}$, all the amorphous gels were found to form through the evaporation of approximately half of the solvent droplet by volume. The background corrected $\mathrm{x}$-ray intensities for amorphous Clotrimazole, Probucol, (racemic) Ibuprofen, Dibucaine, Ketoprofen, and Clofoctol at time $t_{\mathrm{P}}$ are shown in Fig. 3. At, or just prior to, $t_{\mathrm{P}}$ the gels were quenched into a small dish of liquid nitrogen in an attempt to preserve the amorphous forms. The cold solid samples were quickly recovered and relevitated. In situ x-ray structure measurements were then performed on the cold solids as they warmed back to room temperature. The diffraction patterns indicated that Clotrimazole, Ibuprofen, Ketoprofen, and Clofoctol remained amorphous during the exposure to cryogenic temperatures, confirming that the amorphous forms could be stored at low temperature. However, Probucol and Dibucaine repeatedly 
shattered upon immersion in liquid nitrogen and the Dibucaine fragments were found to be crystalline.

We propose that the levitation technique described above offers new routes for processing amorphous molecular materials. This is important in pharmaceutical science because the properties (solubility, dissolution rates) and function (bioavailability) of stable or metastable amorphous gels are often significantly different from their crystalline counterparts [22,23]. Previously, for example, Calcium Ketoprofen dehydrate could only be vitrified by quenching from the melt in liquid nitrogen and is known to crystallize from water-ethanol supersaturated solutions in containers [24]. Similar containerless amorphization behavior is found here for Clotrimazole, Dibucaine, and Clofoctol. All of these molecules crystallize from ethanol solutions in containers but can form glasses by rapid quenching from the melt [15]. In at least two of the cases in this study, namely, Probucol and Ibuprofen, amorphous forms of the drugs have previously been produced by spray drying and mixing with a stabilizing agent. Although Probucol rapidly crystallizes from saturated solutions of ethanol or acetone into one of two polymorphs [25], an amorphous form of the drug has been stabilized by mixing it with a hydrophilic polymer and spray drying [26]. Similarly, Ibuprofen readily crystallizes from an ethanol solution [27], however, a stabilized amorphous form of this drug has also been obtained by cospraying with mesoporous submicron particles to yield an enhanced dissolution rate [28]. Pure Ibuprofen, however, does form a supercooled liquid upon cooling from the melt and will not crystallize provided the temperature remains above $-10^{\circ} \mathrm{C}$ since no nuclei form above this temperature [16]. Consequently, further structure-property characterizations of the amorphous drugs produced by the levitation technique are needed in order to elucidate differences with the same materials amorphized using other techniques and mixtures.

\section{B. Vitrification by laser melting}

It is also demonstrated that pure molecular liquids of pharmaceutical drugs can be vitrified by melting their crystalline forms under containerless conditions in an acoustic levitator. The drugs Cinnarizine, Carbamazepine, Miconazole Nitrate, Probucol, Clofoctol, and Ketoprofen were melted, using 1-5 $\mathrm{W}$ of heating power from an unfocused $10 \mathrm{~W} \mathrm{CO}$ laser beam. The $\sim 2 \mathrm{~mm}$ diameter ( $4 \mu \mathrm{L}$ in volume) levitated droplets were acoustically translated out of the laser beam and cooled in the levitator to form fully or partially glassy solids. It is estimated that $\sim 25 \%$ of the laser beam power was absorbed by the samples depending on alignment and sample size. In all cases, laser heating caused instabilities in the levitation, mainly in the form of radial oscillations of approximately $\pm 2 \mathrm{~mm}$ in magnitude or fast spinning. The liquid droplets generally formed a pancake shape and required a higher acoustic power to levitate than the starting solid forms, indicating a low surface tension. The recovered levitated products were stored in the refrigerator prior to the x-ray experiments. For comparison, the same starting materials (of a similar size) were gently heated with a laser beam in a copper hearth to demonstrate the effect of a container on the samples. All the samples processed in the copper hearth resulted in solid polycrystalline forms. For Cinnarizine, Carbamazepine, and Miconazole Nitrate, the molten droplets obtained by laser heating were fully vitrified when cooled in the acoustic levitator, whereas the corresponding liquids left to cool in containers formed crystalline solids. The example of the color change to yellow glassy Cinnazirine compared to white crystalline Cinnazirine is shown in Fig. 1(b). The yellow discoloration may be due to the cracking of some hydrocarbons in the drug by the laser and NMR experiments are in progress to determine if this is the case.

The x-ray structure factors and corresponding differential distribution functions $D(r)$ [29] for the laser heated samples quenched in the levitator, which were measured 3 days after processing, are shown in Figs. 4 and 5, respectively. The x-ray pair distribution functions were obtained through a sine-Fourier transformation of the $\mathrm{S}(\mathrm{Q})$ curves truncated at positive nodes corresponding to $Q_{\max }=19.4 \AA^{-1}$ using a Lorch modification function [30] to reduce Fourier artifacts.

The Bragg peaks observed in the materials cooled in containers indicate long range ordering in real space, and while the well-defined molecular bonds at low-r persist in the glassy pair distribution functions, the local packing arrangement of molecules is completely broken down. We note that the longest intramolecular distances in Cinnarizine is $16.6 \AA$, in Carbamazepine it is $9.0 \AA$, and

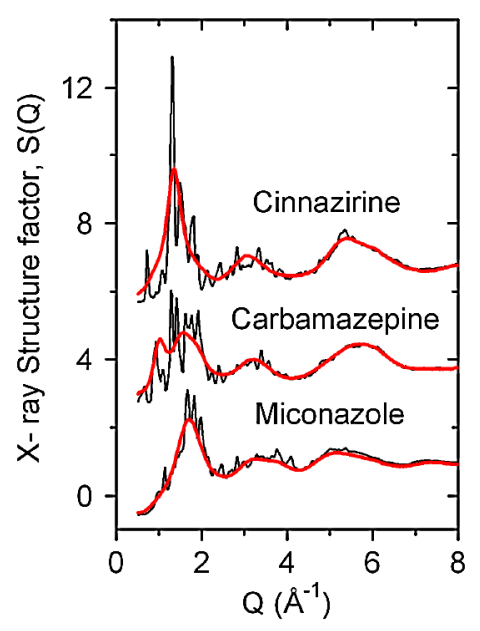

FIG. 4. The X-ray structure factors, $S(Q)$, for three pure pharmaceutical drugs melted in the levitator with a laser and quenched to room temperature (thick red lines). The $S(Q)$ functions are compared to the same starting materials melted and allowed to cool in a copper hearth (thin black lines). 


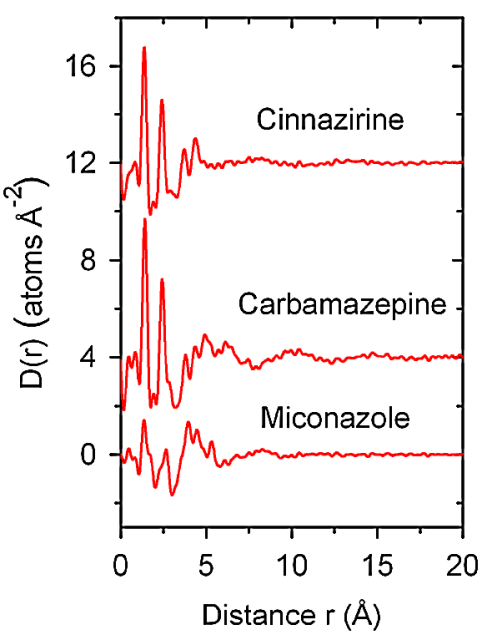

FIG. 5. The x-ray differential pair distribution functions, D(r) [29], corresponding to the $S(Q)$ curves (thick red lines) in Fig. 4, for three pure pharmaceutical drugs melted in the levitator with a laser and quenched to room temperature.

in Miconazole Nitrate it is $13.3 \AA$. Little structure is observed in any of the $\mathrm{D}(\mathrm{r})$ functions beyond $\sim 15 \AA$, indicating a wide distribution of orientational correlations between neighboring molecules in the amorphous forms. Of these drugs, Carbamazepine has been the most widely studied and differential scanning calorimetry measurements have reported difficulty in producing a purely amorphous form, even upon rapid quenching of the melt [15]. This is despite the fact that Carbamazepine has four crystalline polymorphs, all with similar molecular conformations and stabilities, and identical hydrogen bonding patterns [31]. Similar x-ray experiments on pure Probucol, Clofoctol, and Ketoprofen measured one week after laser heated containerless processing resulted in partial vitrification for levitated samples compared to the formation of fully crystalline forms cooled in containers. The melting and glass transition temperatures for the drugs melted in the levitator have been reported by Baird et al. [15]. We note that in the cases of Ketoprofen, Clofoctol, Cinnazirine, and Miconazole Nitrate the glass transition temperature is less than room temperature and the samples were recovered and stored as supercooled liquids for a few days before $x$-ray characterization. This indicates that the kinetics of crystallization are relatively slow for these liquids.

\section{CONCLUSIONS AND FUTURE DIRECTIONS}

In crystalline pharmaceutical solids, relationships between precise structural molecular conformations and arrangements in the crystal structure can be made with chemical reactivity in specific biological interactions. However, little information is known regarding the effect of detailed microscopic amorphous structure and its relation to material properties [32]. At the atomic or molecular level, glassy and amorphous materials may take on a range of structures depending on their preparation history, making absolute characterization difficult [33]. Nonetheless, the results found here from x-ray total pair distribution function measurements of organic pharmaceuticals are in general agreement with previous levitation experiments on deeply supercooled liquids or glassy oxide materials, which show a wide distribution of packing arrangements compared to crystalline analogues $[9,33]$. It is likely that, in the case of pharmaceuticals, the structural differences may be caused by changes in molecular conformation, hydrogen bonding, or differences in other intermolecular forces.

For the experiments described in this letter, no steps were taken to optimize the levitator for amorphization by minimizing dust (which could inadvertently cause crystallization) or mixing the drugs with different stabilizers, which would help to inhibit crystallization during the containerless processing. Future investigations into both these effects and the shelf life of the amorphous materials synthesized by acoustic levitation will be necessary to determine the full implications of this technique for the pharmaceutical industry. This will require the construction of detailed time-temperature-transformation diagrams for liquids processed under containerless conditions compared to those in containers, as has recently been performed in the case of racemic Ibuprofen [16]. So far we have found that melt quenched Cinnazirine and Carbamzepine processed in the acoustic levitator remain completely amorphous for at least 4 months after amorphization but Clofoctol crystallizes within a few weeks and Dibucaine in a few minutes.

We anticipate containerless processing techniques will aid the development of amorphous drug forms, which are becoming increasingly important due to the emergence of new drugs that are virtually insoluble in their crystalline form $[5,6]$. In order to form glass, a liquid must be cooled at a rate greater than or equal to the critical cooling rate, $R_{c}$. It is well established for high temperature melts that $R_{c}$ is usually much smaller when sources of extrinsic nucleation are removed, i.e., under containerless conditions. Small samples are therefore beneficial in vitrifying reluctant glass formers because they allow faster cooling rates. Similarly, the supersaturation of solutions also occurs more readily when surfaces are eliminated. Possible routes for scaling up production might be through the use of drop towers (as used for producing lead shot) or drip cooling methods. In addition, we note that acoustic levitation enables the amorphization process to be characterized in situ which is not practical with falling drop methods.

Future aspects of the work will concentrate on amorphizing reluctant glass formers which have never been vitrified and extending the technique to other areas such as food science and biology [34,35]. Toward this end, we have recently investigated the drug lidocaine 
[2-(diethylamino)- $N$-(2,6-dimethylphenyl)acetamide] [36], which has a melting point of $68^{\circ} \mathrm{C}$ and a glass transition temperature of $-39^{\circ} \mathrm{C}$. The melt of the drug crystallizes as soon as it comes into contact with any external surface. However, it forms an amorphous product in the levitator and is stable at room temperature.

\section{ACKNOWLEDGMENTS}

This work is supported by the U.S. Department of Energy, Basic Energy Sciences, Office of Science, under Contract No. DE-AC02-06CH11357. Professor S. Byrn is thanked for useful discussions and initiating the work on lidocaine. Professor L. Taylor is thanked for a preprint of Ref. [15].

[1] L. Yu, Amorphous Pharmaceutical Solids: Preparation, Characterization, and Stabilization, Adv. Drug Delivery Rev. 48, 27 (2001).

[2] J. F. Willart and M. Descamps, Solid State Amorphization of Pharmaceuticals, Molecular Pharmaceutics 5, 905 (2008).

[3] J. Lu and S. Rohani, Polymorphism and Crystallization of Active Pharmaceutical Ingredients (APIs), Curr. Med. Chem. 16, 884 (2009).

[4] K. Kawakami, Current Status of Amorphous Formulation and Other Special Dosage Forms as Formulations for Early Clinical Phases, J. Pharm. Sci. 98, 2875 (2009).

[5] K. Nagapudi and J. Jona, Amorphous Active Pharmaceutical Ingredients in Preclinical Studies: Preparation, Characterization, and Formulation, Current Bioactive Compounds 4, 213 (2008).

[6] P. Gao, Amorphous Pharmaceutical Solids: Characterization, Stabilization, and Development of Marketable Formulations of Poorly Soluble Drugs with Improved Oral Absorption, Molecular Pharmaceutics 5, 903 (2008).

[7] E.H. Trinh, Compact Acoustic Levitation Device for Studies in Fluid Dynamics and Material Science in the Laboratory and Microgravity, Rev. Sci. Instrum. 56, 2059 (1985).

[8] D.L. Price, High-Temperature Levitated Materials (Cambridge University Press, Cambridge, England, 2010), ISBN 978-0-521-88052-7.

[9] G. N. Greaves et al., Detection of First-Order Liquid/ Liquid Phase Transitions in Yttrium Oxide-Aluminum Oxide Melts, Science 322, 566 (2008).

[10] J. A. Tangeman, B. L. Phillips, A. Navrotsky, J. K. R. Weber, A. D. Hixson, and T.S. Key, Vitreous Forsterite $\left(\mathrm{Mg}_{2} \mathrm{SiO}_{4}\right)$ : Synthesis, Structure, and Thermochemistry, Geophys. Res. Lett. 28, 2517 (2001).

[11] S. Bates, G. Zografi, D. Engers, K. Morris, K. Crowley, and A. Newman, Analysis of Amorphous and Nanocrystalline Solids from Their X-Ray Diffraction Patterns, Pharm. Res. 23, 2333 (2006).

[12] U. Varanasi, R.E. Apfel, and D.C. Malins, A Novel Microtechnique for the Measurement of Acoustic Properties of Lipids, Chem. Phys. Lipids 19, 179 (1977).
[13] S. K. Chung and E.H. Trinh, Containerless Protein Crystal Growth in Rotating Levitated Drops, J. Cryst. Growth 194, 384 (1998).

[14] J. Leiterer, F. Delissen, F. Emmerling, A. F. Thunemann, and U. Panne, Structure Analysis Using Acoustically Levitated Droplets, Anal. Bioanal. Chem. 391, 1221 (2008).

[15] J. A. Baird, B. Van Eerdenbrugh, and L. S. Taylor, A Classification System to Assess the Crystallization Tendency of Organic Molecules from Undercooled Melts, J. Pharm. Sci. 99, 3787 (2010).

[16] E. Dudognon, F. Danede, M. Descamps, and N. T. Correia, Evidence for a New Crystalline Phase of Racemic Ibuprofen, Pharm. Res. 25, 2853 (2008).

[17] J. K. R. Weber, C.A. Rey, J. Neuefeind, and C.J. Benmore, Acoustic Levitator for Structure Measurements on Low Temperature Liquid Droplets, Rev. Sci. Instrum. 80, 083904 (2009).

[18] J. Neuefeind, C. J. Benmore, J. K. R. Weber, and D. Paschek, More Accurate X-Ray Scattering Data of Deeply Supercooled Bulk Liquid Water, Mol. Phys. 109, 279 (2011).

[19] A. P. Hammersley, S. O. Svensson, M. Hanfland, A. N. Fitch, and D. Hausermann, Two-Dimensional Detector Software: From Real Detector to Idealised Image or Two-Theta Scan, High Press. Res. 14, 235 (1996).

[20] X. Y. Qiu, J. W. Thompson, S. J. L. Billinge, PDFgetX2: A GUI-Driven Program to Obtain the Pair Distribution Function from X-Ray Powder Diffraction Data, J. Appl. Crystallogr. 37, 678 (2004).

[21] C. J. Benmore and Y.L. Loh, The Structure of Liquid Ethanol: A Neutron Diffraction and Molecular Dynamics Study, J. Chem. Phys. 112, 5877 (2000).

[22] F. Franks, Solid Aqueous Solutions, Pure Appl. Chem. 65, 2527 (1993).

[23] G. W. Scherer, Aging and Drying of Gels, J. Non Cryst. Solids 100, 77 (1988).

[24] F. Atassi, C. Mao, A. S. Masadeh, and S. R. Byrn, Solid State Characterization of Amorphous and Mesomorphous Calcium Ketoprofen, J. Pharm. Sci. 99, 3684 (2010).

[25] J. J. Gerber, M. R. Caira, and A.P. Lotter, Structures of Two Conformational Polymorphs of the CholesterolLowering Drug Probucol, J. Crystallogr. Spectrosc. Res. 23, 863 (1993).

[26] P. Thybo, B. L. Pedersen, L. Hovgaard, R. Holm, and A. Mullertz, Characterization and Physical Stability of Spray Dried Solid Dispersions of Probucol and PVP-K30, Pharm. Dev. Technol. 13, 375 (2008).

[27] H. Cano, N. Gabas, J. P. Canselier, Experimental Study on the Ibuprofen Crystal Growth Morphology in Solution, J. Cryst. Growth 224, 335 (2001).

[28] S.-C. Shen, W. K. Ng, L. Chia, Y. C. Dong, and R. B. Tan, Stabilized Amorphous State of Ibuprofen by Co-Spray Drying with Mesoporous SBA-15 to Enhance Dissolution Properties, J. Pharm. Sci. 99, 1997 (2010).

[29] D. A. Keen, A Comparison of Various Commonly Used Correlation Functions for Describing Total Scattering, J. Appl. Crystallogr. 34, 172 (2001).

[30] E. A. Lorch, Neutron Diffraction by Germania, Silica, and Radiation-Damaged Silica Glasses, J. Phys. C 2, 229 (1969). 
[31] A. I. Grzesiak, L. Meidong, K. Kim, and A. J. Matzger, Comparison of the Four Anhydrous Polymorphs of Carbamazepine and the Crystal Structure of Form I, J. Pharm. Sci. 92, 2260 (2003).

[32] E. Shalaev and G. Zografi, in Amorphous Food and Pharmaceutical Systems, edited by H. Levine (The Royal Society of Chemistry, Cambridge, 2002), pp. 1130, ISBN 0-85404-866-9.

[33] C. A. Tulk, C. J. Benmore, J. Urquidi, D. D. Klug, J. Neuefeind, B. Tomberli, and P. A. Egelstaff, Structural
Studies of Several Distinct Metastable Forms of Amorphous Ice, Science 297, 1320 (2002).

[34] C. A. Angell, R. D. Bressel, J. L. Green, H. Kanno, M. Oguni, and E.J. Sare, Liquid Fragility and the Glass Transition in Water and Aqueous Solutions, J. Food Eng. 22, 115 (1994).

[35] C. A. Angell, Formation of Glasses from Liquids and Biopolymers, Science 267, 1924 (1995).

[36] A. Tailor, Z. Su, X. Chen, J. K. R. Weber, C. J. Benmore, L. Taylor, and S. R. Byrn (unpublished). 